\title{
Aggression in ADHD: relation to salivary cortisol
}

\author{
Reem H. El Ghamry'1, Mona M. Mohamed', Hanan M. Azzam¹, Mahmoud M. Elhabiby', Haytham M. Hasan²,
} Adel F. Hashish ${ }^{3}$, Mohammed M. Elhamshary ${ }^{4}$ and Doaa M. H. Barakat ${ }^{1^{*}}$ (D)

\begin{abstract}
Background: Study of cortisol levels in patients with ADHD in correlation with aggressive behaviors associated with ADHD has received limited research attention. This factor is essential for comprehending the psychopathophysiology of ADHD and its comorbidities. The present study aimed to investigate the cortisol level in ADHD children and its relation to severity of symptoms and associated aggressive behavior in those children. The sample consisted of 129 patients and 80 healthy controls evaluated by administering the Mini International Neuropsychiatric Interview for Children, The Wechsler Intelligence Scale for Children, Conners' parent rating scale, problem scale of C.B.C.L., and Socioeconomic Status Scale. Salivary cortisol was measured using radio-immune assay.

Results: Salivary cortisol level in our ADHD subjects was significantly lower $(11.826 \mathrm{ng} / \mathrm{ml})$ than in the control group (19.619 $\mathrm{ng} / \mathrm{ml}$ with $P$-value 0.001). Our results failed to find any correlation between ADHD symptoms severity and salivary cortisol levels. Severity of delinquent, aggressive, and externalizing behaviors of ADHD children positively correlated with cortisol salivary levels.

Conclusion: Salivary cortisol levels are lower in children with ADHD relative to age- and sex-matched healthy controls. In addition, there is no obvious correlation between severity of ADHD symptoms and basal salivary cortisol levels. We also concluded that there is positive correlation between delinquent, aggressive, and externalizing behaviors in children with ADHD and their basal salivary cortisol levels.
\end{abstract}

Keywords: ADHD, Aggression, Salivary cortisol, Attention deficit, Hyperactivity

\section{Background}

It is widely accepted that the cause of attention-deficit hyperactivity disorder (ADHD) is multifactorial, including both biological and environmental factors. One of the biological determinants of ADHD is the hypothalamic-pituitaryadrenal (HPA) axis dysregulation. Low basal cortisol levels and abnormal diurnal cortisol rhythms have been reported in patients with ADHD. The low levels may be attributed to the under-arousal probably causing several of the key symptoms of ADHD [1].

\footnotetext{
*Correspondence: doaab@hotmail.com

'Okasha Institute of Psychiatry, Faculty of Medicine, Ain Shams University, 22 Dair Almalak, Abbassia, Cairo 11657, Egypt

Full list of author information is available at the end of the article
}

A diversity of experiences may lead to childhood aggression. Accumulating experimental evidence indicates that stress in early life is a major risk factor for the development of pathological aggression [2]. Stress in early life can happen in several ways such as early social deprivation or being exposed to neglect, trauma, or abuse. Moreover, impaired social skills have been related to childhood aggression as well; children that are unable to adapt to their social environment can behave in an aggressive way [3]. Structural and functional variations in the brains of people subjected to maltreatment have been identified in several studies [4]. Neuroendocrinal disturbance is one of the biological determinants of childhood aggression. Lower levels of basal cortisol were found in longitudinal studies as a

\section{Springer Open}

(c) The Author(s). 2021 Open Access This article is licensed under a Creative Commons Attribution 4.0 International License, which permits use, sharing, adaptation, distribution and reproduction in any medium or format, as long as you give appropriate credit to the original author(s) and the source, provide a link to the Creative Commons licence, and indicate if changes were made. The images or other third party material in this article are included in the article's Creative Commons licence, unless indicated otherwise in a credit line to the material. If material is not included in the article's Creative Commons licence and your intended use is not permitted by statutory regulation or exceeds the permitted use, you will need to obtain permission directly from the copyright holder. To view a copy of this licence, visit http://creativecommons.org/licenses/by/4.0/. 
predictor of aggression or as a marker for severe and continuous aggression [5-7].

McBurnett et al. [7] studied the association between plasma cortisol levels and aggression in children aged 7 to 12 and found that low cortisol levels were linked to aggressive patterns and early onset of aggression in male children.

Callous-unemotional traits are believed to increase risk for aggression through diminished amygdala responsiveness to distress cues. Low cortisol reactivity is thought to increase the likelihood of aggression by lowering arousal, but this effect can only be seen in boys. Cortisol reactivity moderated the relationship between $\mathrm{CU}$ traits and aggression, with a significant association in the presence of low cortisol reactivity and a minor and nonsignificant association in the presence of high cortisol reactivity. The combination of a deficiency in inhibitory processes linked to $\mathrm{CU}$ characteristics and low cortisol reactivity raises the risk of childhood aggression, with this effect predominantly affecting boys [8].

Study of cortisol levels in patients with ADHD in correlation with aggressive behaviors associated with ADHD has received limited research attention. The present study aimed to correlate basal cortisol levels in ADHD children to severity of their symptoms and to severity of their associated aggressive behaviors. We hypothesized that cortisol levels in children with ADHD correlated to severity of the aggressive behavior associated with ADHD.

\section{Methods}

\section{Study design and site of the study}

This is a cross-sectional comparative study. We recruited cases from the Child Psychiatry Clinic, Okasha Institute of Psychiatry, Ain-Shams University Hospitals.

\section{Participants}

The sample consisted of a patient group and a control group. The number of required cases was estimated by an expert statistician (using Epi Info program) according to power of calculation with statistical power of $80 \%$ and statistical significance defined at 0.05 [9] and based on similar studies carried out by McBurnett et al. [7] and Kariyawasam et al. [6]. The control group was a convenient sample recruited during the period of the study conduction.

Participants were recruited over a period of 6 months from January to June 2017. One hundred fifty-two patients and 92 controls were examined for eligibility; out of these, 140 patients and 86 controls were confirmed eligible; the salivary cortisol samples of 11 patients and 6 controls either got lost or damaged in the lab, and hence, they were excluded from the study, leaving us with 129 patients and 80 controls, with 0.6 controls per case.
The patient group consisted of newly diagnosed unmedicated children with ADHD-according to DSM-IVTR-ranging from 6 to 12 years old of both sexes. Exclusion criteria were (i) below average IQ, (ii) presence of comorbid psychiatric disorders rather than disruptive behavior disorders, (iii) presence of chronic medical illness, and (iv) receiving steroid-containing medications. The children in the control group were healthy and gender- and age-matched children selected from siblings of patients attending the clinic.

\section{Procedures}

After receiving the approval of Faculty of Medicine, Ain Shams University Research Ethics Committee under license number FMASU 1473/2013, the study proper was carried as described below:

1. We obtained a written informed consent from the guardians of both patients and controls involved in the study.

2. The guardians of children of both groups completed the socioeconomic status scale. It consists of 7 domains: Family, Economic, Occupation, Health care, Education and cultural, Family possessions, and Home sanitation. The total score is 84 with a higher score indicating better socioeconomic status. Scores were categorized into very low, low, middle, and high levels [10].

3. Each child of the patient group as well as the control group was subjected to the following:

i) Detailed history taking and full general examination for exclusion of any medical condition that might interfere with the process of the study.

ii) IQ was assessed using the Wechsler Intelligence Scale for Children-Arabic version (WISC) to exclude children with below average IQ. It assesses the cognitive and intellectual capabilities. It comprises 6 verbal and 5 performance subsets. Scoring comprises performance IQ, verbal IQ, and total IQ $[11,12]$.

iii) Psychiatric assessment using the Mini International Neuropsychiatric Interview for Children-Arabic version (M.I.N.I. Kid) to exclude any psychiatric morbidity in the control group and to confirm the ADHD diagnosis and exclude psychiatric comorbidities rather than disruptive behavior disorders in the patient group. It involves closed-ended questions reframing the ICD-10 and DSM-IV criteria $[13,14]$.

iv) A sample of saliva was obtained at a fixed time (9 $\mathrm{am}$ ) in both groups to estimate single-point concentrations of salivary cortisol. Estimation of cortisol level in saliva was done using standardized 
radioimmunoassay technique in the labs of National Research Centre, Cairo.

4. The Conners' Parent Rating Scale-Revised-Arabic version; Long Version (CPRS-R-L) was completed by guardians of children in patient group for assessment of the severity of ADHD symptoms. The questionnaire consists of eighty items scoring the parents' description of their child's behavior during the previous month $[15,16]$.

5. The Arabic version of the problem scale of the Child Behavior Checklist for ages 4-18 (CBCL 418) was completed by the guardians of children in patient group for assessment of the associated aggressive and externalizing behaviors. In the current study, we used only the problem scale which is a questionnaire of one hundred and eighteen items. The questions are divided into nine dimensions which are summated altogether to give (1) externalizing problem and (2) internalizing scores and a (3) total score [16, 17].

6. Statistical analysis was done using the Statistical Package for Social Sciences (SPSS), version 16. Results were tabularized, assembled, and statistically analyzed using mean and standard deviation (SD) for parametric numerical (quantitative) data, and Spearman correlation test $(r)$ for assessing the correlation (direction and power) of quantitative variables. A $p$ value of $<0.05$ was deemed significant statistically.

\section{Results}

\section{Demographic data}

The clinical sample of this study consisted of two groups; a case group consisting of 129 children diagnosed as having ADHD according to DSM-IV-TR and a control group consisting of 80 age- and sex-matched healthy children. Ninety-nine children (76.75\%) of the ADHD group were males and 30 were females (23.25\%); meanwhile, 58 children (72.5\%) of the control group were males and 22 children (27.5\%) were females. There was no statistically significant difference between the two groups regarding gender distribution $(p$ value $=0.49)$.

The mean age of the ADHD group was $8.21( \pm 2.4)$ and the mean age of the control group was $8.5( \pm 1.2)$. There was no statistically significant difference between the two groups regarding age ( $p$ value $=0.2$ )

As regards socioeconomic class, $5(3.875 \%)$ of the ADHD group were of very low socioeconomic class, 68 (52.713\%) were of low class, $56(43.41 \%)$ children were of moderate class, and no one of the children belonged to high socioeconomic class. Regarding the control group, 5 (6.2\%) were of very low socioeconomic class, 40
(50\%) were of low class, $32(40 \%)$ children were of moderate class, and only $3(3.8 \%)$ of the children belonged to high socioeconomic class. There was no statistically significant difference between the two groups regarding socioeconomic class $(p$ value $=0.13$ ).

\section{ADHD symptoms' profile in the case group}

Assessment of ADHD symptoms in the case group was done using the Arabic Conner's Parent Rating Scale; long version revealed that there were significant problems in the following domains: Oppositional (mean score $=70$ ); Inattention (mean score $=79.5$ ); Hyperactivity (mean score $=76.5$ ); ADHD index (mean score $=77.8$ ); global index: Restless-Impulsive (mean score $=73.8$ ); global index: Emotional liability (mean score $=73.2$ ); global index: total (mean score $=74.6$ ); DSM-IV: Inattentive (mean score $=79.3)$; DSM-IV: Hyperactivity-Impulsive (mean score $=74.3$ ); and DSM-IV: Total score (mean score $=73.8$ ). Regarding the social problems domain, there were just mild problems which indicate mild affection of self-esteem and confidence as well as affection of relationship with peers. Meanwhile, the Anxiety-Shyness, Perfectionism, and Psychosomatic domains were within normal ranges.

\section{Behavioral problems in children with ADHD}

Assessment of behavioral problems in children with ADHD using the problem scale of the Arabic version of Child Behavior Checklist (CBCL) for ages 4-18 revealed the presence of significant attention problems (mean score $=72.52$ ), significant externalizing problems (mean score $=65.78)$ which indicates significant presence of negative behaviors that are directed toward the external environment, and borderline range of aggressive behaviors $($ mean score $=67.02)$.

\section{Levels of salivary cortisol in children with ADHD and healthy controls}

- Comparison between children with ADHD and healthy children regarding levels of salivary cortisol revealed that children with ADHD exhibit lower levels of salivary cortisol $(11.826 \pm 11.5655)$ than in healthy controls (19.619 \pm 14.3706$)$. This difference of levels of salivary cortisol is statistically significant as shown in Table 1.

\section{Correlation between salivary cortisol level and severity of ADHD symptoms}

On examining the correlation between salivary cortisol levels and severity of ADHD symptoms in the ADHD group as assessed by Conner's Parent Rating Scale, there was no statistically significant correlation between 
Table 1 Comparison of levels of salivary cortisol between children with ADHD and healthy controls

\begin{tabular}{|c|c|c|c|c|c|c|}
\hline & \multicolumn{2}{|c|}{$\begin{array}{l}\text { Cases } \\
n .=129\end{array}$} & \multicolumn{2}{|c|}{$\begin{array}{l}\text { Control } \\
n .=80\end{array}$} & \multirow[t]{2}{*}{$\begin{array}{l}P \\
\text { value }\end{array}$} & \multirow[t]{2}{*}{ Significance } \\
\hline & Mean & SD & Mean & SD & & \\
\hline Salivary cortisol levels & 11.826 & 11.5655 & 19.619 & 14.3706 & 0.001 & Significant \\
\hline
\end{tabular}

salivary cortisol levels and any of the 14 domains of Conner's Parent Rating Scale as shown in Table 2.

\section{Correlation between salivary cortisol level and behavioral problems in children with ADHD}

- On examining the correlation between salivary cortisol levels and behavioral problems in the ADHD group as assessed by the problem scale of the child behavior checklist for ages 4-18, there was statistically significant correlation between salivary cortisol levels and delinquent behavior, aggressive behavior, and externalizing problems as shown in Table 3.

\section{Discussion}

The current study was designed to explore the cortisol level in ADHD children and their correlates to severity of symptoms and associated aggressive behaviors in those children.

The data about salivary cortisol reported in the current study indicates that ADHD subjects have statistically significant lower salivary cortisol $(11.826 \mathrm{ng} / \mathrm{ml})$ level when compared to age- and sex-matched control subjects (19.619 ng/ ml with $P$-value 0.001). This finding is controversial in different studies and we will elaborate some examples of those studies. Our results failed to find any correlation between severity of ADHD symptoms in the children of the case group with their salivary cortisol levels.

Three distinct reasons for the low levels of cortisol in ADHD children can hypothetically be established. First, they may demonstrate a diurnal curve that is displaced, indicating that at the normal time of the morning peak, maximum levels have either been passed or not reached. There is some support from a small-scale (no.=13) research on adults for such a theory, indicating a phase delay in people with ADHD [18]. In our study, the low morning levels can be explained by a late morning peak.

Second, an increase in the secretion of glucocorticoids due to environmental factors for example early childhood psychosocial adversity or perinatal stress is likely to have an adverse impact on the development of brain networks in genetically susceptible children, which may explain the dysregulation of the HPA axis resulting in deficits in arousal regulation and the development of externalizing symptoms of ADHD as a result. Third, certain genetic or environmental circumstances may lead to ADHD symptoms as well as low activity of the HPA axis [19].

Studies with similar results included Susman et al. [20] who originally observed a normal diurnal pattern of cortisol in only $43 \%$ of ADHD children, indicating that in most children with ADHD the HPA axis is dysregulated. In other studies, cortisol awakening response was found

Table 2 Correlation between salivary cortisol levels and scores of Conner's Parent Rating Scale in the ADHD group

\begin{tabular}{llll}
\hline Correlation between Conners' domains with salivary cortisol levels & $\boldsymbol{R}$ value & $\boldsymbol{P}$ value & Significance \\
\hline Opposition & 0.047 & 0.61 & Non-significant \\
Inattention & -0.039 & 0.68 & Non-significant \\
Hyperactivity & 0.109 & 0.24 & Non-significant \\
Anxiety-Shyness & -0.116 & 0.22 & Non-significant \\
Perfectionism & -0.09 & 0.34 & Non-significant \\
Social problems & -0.005 & 0.96 & Non-significant \\
Psychosomatic & -0.073 & 0.44 & Non-significant \\
Conners' ADHD index & -.031 & 0.74 & Non-significant \\
Conners' index: Restless-Impulsive & 0.108 & 0.25 & Non-significant \\
Conners' index: Emotional lability & 0.061 & 0.51 & Non-significant \\
Conners' index: total & 0.076 & 0.41 & Non-significant \\
DSM-IV: Inattentive & 0.000 & 0.99 & Non-significant \\
DSM-IV: Hyperactivity-Impulsive & 0.102 & 0.28 & Non-significant \\
DSM-IV: Total & 0.111 & 0.23 & Non-significant \\
\hline
\end{tabular}


Table 3 Correlation between salivary cortisol level and behavior problems assessed by CBCL in the ADHD group

\begin{tabular}{llll}
\hline Correlation between CBCL (Problem scale) with salivary cortisol levels & $\boldsymbol{R}$ value & $\boldsymbol{P}$ value & Significance \\
\hline Delinquent behavior & 0.173 & 0.049 & Significant \\
Aggressive behavior & 0.226 & 0.01 & Significant \\
Externalizing problems & 0.186 & 0.035 & Significant \\
\hline
\end{tabular}

to be lower in ADHD children when compared to a control group [21, 22].

A study by Isaksson and his group in 2012 found that compared to non-affected comparisons, children with ADHD had significantly lower levels of salivary cortisol. ADHD drugs and gender did not affect the cortisol levels. Cortisol levels were not affected by the cooccurring symptoms or the ADHD subtype within their study population. The symptoms of ADHD severity degree in the study group was not correlated with cortisol levels [1].

Isaksson and colleagues [23] tested salivary cortisol in 197 ADHD children and 221 controls using radioimmunoassay in a subsequent study. They found that salivary cortisol levels were considerably lower in ADHD children relative to the control group at awakening and 30 min after awakening. This may be because there is a distorted circadian rhythm associated with ADHD. A different finding to ours is that a negative correlation was documented between symptoms of ADHD and levels of cortisol at awakening and $30 \mathrm{~min}$ after awakening [23].

In accordance with our results, Hastings and colleagues [24] did not find any association between symptoms of ADHD and awakening levels of cortisol in their analysis of salivary cortisol in 170 ADHD children.

In addition, a recent Egyptian study comparing morning and bedtime salivary cortisol showed that the levels of cortisol in samples collected at bedtime of patients with ADHD were lower significantly compared to those of the control group with no statistically significant difference detected at the morning sample [25].

Hypothetically, a correlation between a HPA axis that is downregulated and ADHD fits hypotheses that consider ADHD as a consequence of under-arousal. The optimal stimulation hypothesis is one such theory, which indicates that ADHD presentations may be considered as manifestations of over activity aimed at stimulating arousal for example through taking risks, aggressive behavior, talking, attention shifting, or stimulation seeking [1].

Three related neuropsychological mechanisms were proposed: fight or flight, sensitivity to reward (facilitated through "behavioral activation system" the BAS), and sensitivity to punishment (facilitated through "behavioral inhibition system" the BIS). Individuals vary in accordance to this theory: some are usually inclined to seek incentives (high BAS), while others are highly motivated to resist punishing stimuli (high BIS). BIS activity triggers the termination of recurrent behavior, focuses the attention of the organism on environmental cues, and raises the physiological unspecific arousal [26].

It is argued that BIS functional impairment contributes to hyperactivity/impulsivity symptomatology of ADHD in addition to neurocognitive deficits in motor fluency, control of emotions and verbal and visual working memory [27].

Low cortisol levels in ADHD whether at baseline or in conditions of psychological stress can be due to an underactive BIS. It is proposed that impaired BIS activity is rather unique for the predominantly hyperactive/impulsive subtype or the combined subtype of ADHD [27]. One hypothesis arising from this theory is that in these two subtypes, but not in the primarily inattentive form of ADHD, abnormalities should be observed in HPA axis activity.

Other studies have described more levels of morning cortisol in ADHD children, unlike our research [28], and in boys with hyperactivity/impulsivity [29], while other research failed to detect any variation in baseline levels of cortisol between ADHD participants and healthy controls [30-32].

The daily variation of the secretion of cortisol is characterized by the high waking levels, an increase further in the morning and a gradual decrease in the day till midnight [33]. The sampling timing may, therefore, be responsible for the different results between studies. The different results could also be due to different cortisol measuring techniques, saliva collection protocols, or variation within the participants with regard to comorbid disorders. In our study, we controlled for the time of sample collection and comorbid disorders apart from disruptive behavior disorders.

In the current study, the severity of delinquent, aggressive, and externalizing behaviors of ADHD children were positively correlated with salivary cortisol levels. A recent British study reported similar findings with a positive correlation between oppositional defiant and conduct symptoms in ADHD children with their salivary cortisol levels [34].

One of the studies examining the relationship between ADHD, disruptive behavior disorders, and cortisol was that conducted by Kariyawasam et al. [6]. Compared to 25 healthy controls of similar age and ethnic background, salivary cortisol was determined in thirty-two children with ADHD and comorbid oppositional defiant 
disorder. Salivary cortisol was lower significantly in the ADHD/oppositional defiant comorbid group compared to the control group.

If the low activity of the HPA axis and symptoms of ADHD do in fact stem from common neural deviations, low activity of the HPA axis may show this deviation at an early phase and may therefore predict the development of ADHD symptoms in the preschool years. Low activity of the HPA axis may be viewed as an earlier predictor of the progression of the disorder if this is the case [18]. Indeed, Salis et al. [35] found that at age 6, a diminished diurnal salivary cortisol profile indicated a rise in externalizing symptoms till age 9 .

Schloß et al. in 2018 found that at the age of 4 years low hair cortisol concentration, that is, a low HPA axis activity incorporated over a period of 3 months, anticipated increased symptoms of ADHD in the next 12 months, which was statistically significant only in the boys not in girls [19].

A 2018 study explored serum cortisol level in children with attention-deficit/hyperactivity disorder combined subtype and another group of children with ADHD combined subtype and comorbid conduct disorder. Among the study groups, no substantial difference in serum cortisol levels was observed. A negative correlation was also found between serum cortisol levels and oppositional defiant behavior scores [36].

Cortisol awakening response (CAR) is the rise in levels of cortisol of about $50 \%$ happening in some individuals 20 to 30 min following morning awakening. A study in 2009 found a blunted CAR in ADHD children with comorbid oppositional defiant disorder compared to ADHD children with comorbid conduct disorder, ADHD with no comorbidity, and healthy controls [37].

One study measured the diurnal pattern of cortisol in a group of thirty ADHD children, compared to a psychiatric control group consisting of twenty-one children with autism and a control group of adults. Samples of saliva were collected in the morning at 2-h periods. More samples were collected in the afternoon and evening to illustrate the diurnal pattern. The authors found that most of the children with ADHD (57\%) did not exhibit a regular diurnal pattern, while diurnal rhythm abnormalities were found in only $10 \%$ of adult controls and $20 \%$ of autistic subjects [38].

It was found in a study of male children between 10 and 12 years of age that low levels of cortisol were associated with impulsivity and aggression after 5 years [39]. Lopez-Duran et al. [40] evaluated cortisol reactivity in seventy-three 6 to 7 -year-old children and found that cortisol reactivity was higher in those with forms of reactive aggression than in children with proactive aggression or those without aggression [40]. Furthermore, another study in 2005 found that low levels of basal cortisol were correlated with callous traits in male adolescents, but this association was not valid in female adolescents. Serum concentrations of cortisol can be recognized as "emotional reactivity" biomarkers, and therefore, cortisol levels can potentially aid in predicting the type of aggression and contribute with diagnosis and treatment [41].

Consideration of the etiology of HPA pathology and pediatric aggression is important. Considerable research has to be done in the future to the disturbed cortisol pattern in ADHD as a biomarker to support ADHD diagnosis or to identify certain specifiers.

\section{Conclusions}

Children with ADHD exhibit low levels of basal salivary cortisol in comparison to age- and sex-matched healthy controls. There is no obvious correlation between severity of ADHD symptoms and basal salivary cortisol levels. There is positive correlation between delinquent, aggressive, and externalizing behaviors in children with ADHD and their basal salivary cortisol levels.

\section{Research recommendations and clinical implications}

- Aggression is a common symptom associated with ADHD and needs to be clinically assessed and considered while tailoring a management plan.

- ADHD has biological, social, and psychological determinants. Future research would benefit from integrating a biopsychosocial model in studying ADHD.

- Considerable research must be done in the future regarding disturbed cortisol pattern in ADHD as a biomarker to support ADHD diagnosis or to identify certain specifiers.

\section{Limitations}

- Randomization sampling technique would have decreased selection bias.

\section{Generalizability}

Selecting the sample from clinic referred patients may affect the generalizability of our results to the populationbased cases as most of cases attending public clinics suffer severe symptoms.

\section{Abbreviations}

ADHD: Attention-deficit hyperactivity disorder; BAS: Behavioral activation system; BIS: Behavioral inhibition system; CAR: Cortisol awakening response; CBCL: Child Behavior Checklist for ages 4-18; CPRS-R-L: The Conners' Parent Rating Scale-Revised; Long Version; HPA: Hypothalamic-pituitary-adrenal axis; M.I.N.I. Kid: Mini International Neuropsychiatric Interview for Children-Arabic version; SPSS: Statistical Package for Social Sciences; WISC: Wechsler Intelligence Scale for Children-Arabic version 


\section{Acknowledgements}

The authors would like to express their gratitude for all the participating subjects in this study.

\section{Authors' contributions}

RE: acquisition of data, analysis and interpretation of data, revised the article. MM: came up with the research idea, designed the study, revised the article, final approval of the version to be published, responsibility for the integrity of the work as a whole. HA: came up with the research idea, designed the study, revised the article, final approval of the version to be published, responsibility for the integrity of the work as a whole.ME: acquisition of data, analysis and interpretation of data, revised the article. $\mathrm{HH}$ : acquisition of data, helped in writing the article, revised the article. $\mathrm{AH}$ : came up with the research idea, acquisition of data, analysis and interpretation of data, helped in writing the article. ME: acquisition of data, did the laboratory part of the study, helped in writing the article. DB: acquisition of data, wrote the article, revised the article, responsibility for the integrity of the work as a whole.All authors have read and approved the manuscript.

\section{Funding}

No funding was received for this study.

\section{Availability of data and materials}

The data used in the study is available from the corresponding author upon reasonable request.

\section{Declarations}

\section{Ethics approval and consent to participate:}

Authors received the approval of Faculty of Medicine, Ain Shams University Research Ethics Committee under license number FMASU 1473/2013. A written informed consent was obtained from the guardians of both patients and controls involved in the study.

\section{Consent for publication}

Not applicable.

\section{Competing interests}

All authors declare no financial or non-financial competing interests.

\section{Author details}

'Okasha Institute of Psychiatry, Faculty of Medicine, Ain Shams University, 22 Dair Almalak, Abbassia, Cairo 11657, Egypt. ${ }^{2}$ Faculty of Medicine, Helwan University, Helwan, Egypt. ${ }^{3}$ Centre of Excellence of Medical Research, Medical Division, National Research Centre, Cairo, Egypt. ${ }^{4}$ Northumberland, Tyne and Wear NHS Trust: Cumbria, Northumberland, Tyne and Wear NHS Foundation Trust, Newcastle upon Tyne, UK.

\section{Received: 25 February 2021 Accepted: 30 March 2021}

Published online: 12 May 2021

\section{References}

1. Isaksson J, Nilsson KW, Nyberg F, Hogmark §, Lindblad F (2012) Cortisol levels in children with attention-deficit/ hyperactivity disorder. Journal of psychiatric research 46(11):1398-1405. https://doi.org/10.1016/j.jpsychires.2 012.08 .021

2. Veenema $\mathrm{AH}$ (2009) Early life stress, the development of aggression and neuroendocrine and neurobiological correlates: what can we learn from animal models. Front Neuroendocrinol 30(4):497-518. https://doi.org/10.101 6/j.yfrne.2009.03.003

3. McCrory E, De Brito SA, Viding E (2010) Research review: the neurobiology and genetics of maltreatment and adversity. Journal of Child Psychology and Psychiatry 51(10):1079-1095. https://doi.org/10.1111/j.1469-7610.2010. 02271.x

4. Nemeroff CB (2016) Paradise lost: the neurobiological and clinical consequences of child abuse and neglect. Neuron 89(5):892-909. https:// doi.org/10.1016/j.neuron.2016.01.019

5. Alink LR, Cicchetti D, Kim J et al (2012) Longitudinal associations among child maltreatment, social functioning, and cortisol regulation. Developmental Psychology 48(1):224-236. https://doi.org/10.1037/a0024892
6. Kariyawasam SH, Zaw F, Handley SL (2002) Reduced salivary cortisol in children with comorbid Attention deficit hyperactivity disorder and oppositional defiant disorder. Neuro Endocrinol Lett 23(1):45-48

7. McBurnett K, Lahey BB, Rathouz PJ et al (2000). Low salivary cortisol and persistent aggression in boys referred for disruptive behavior. Arch Gen Psychiatry 57: 38-43.

8. Wright N, Hill J, Pickles A, Sharp H (2019) Callous-unemotional traits, low cortisol reactivity and physical aggression in children: findings from the Wirral Child Health and Development Study. Transl Psychiatry. 9(1):79. https://doi.org/10.1038/s41398-019-0406-9

9. Centers for Disease Control and Prevention. Epi Info. Available at: https:// www.cdc.gov/epiinfo/index.html. [Accessed 20 Nov 2020].

10. El-Gilany A, El-Wehady A, El-Wasify M (2012) Updating and validation of the socioeconomic status scale for health research in Egypt. East Mediterr Health J 18(9):962-968. https://doi.org/10.26719/2012.18.9.962

11. Wechsler DI (1991) Examiner's manual. In: Wechsler intelligence scale for children, 3rd edn. The Psychological Corporation, San Antonio, TX

12. Ismaiel E, Kamel M (1999) Wechsler intelligence scale for children revised, Arabic version, 7th edn. El-Nahda El Massryia, Cairo

13. Sheehan DV, Lecrubier Y, Sheehan KH et al (1998) The Mini-International Neuropsychiatric Interview (M.I.N.I.): the development and validation of a structured diagnostic psychiatric interview for DSM-IV and ICD-10. J Clin Psychiatry 59(20):22-33

14. Ghanem MH, Ibrahim M, El-Behairy AA, El-Merghany H (1999) Mini International Neuropsychiatric Interview for Children / adolescents (M.I.N.I. Kid); Arabic version (1st edition). Department of Neuropsychiatry, Faculty of Medicine, Ain-Shams University, Cairo

15. Conners CK (1997) Conners' Rating Scales-Revised Technical Manual. Multi Health Systems, North Tonawanda, NY

16. El-Sheikh MM, Amer AS, Omar AM and El-Nahas GM (2003). Psychiatric morbidity in first degree relatives of a sample of ADHD children. PhD thesis, Institute of Psychiatry, Ain Shams University, Cairo, Egypt.

17. Achenbach T. Manual for the child behavior checklist / 4-18 and 1991 profiles (1991). Burlington VT; University of Vermont, USA. Department of psychiatry.

18. Baird AL, Coogan AN, Siddiqui A, Donev RM, Thome J (2012) Adult attention-deficit hyperactivity disorder is associated with alterations in circadian rhythms at the behavioural, endocrine and molecular levels. Mo Psychiatry 17(10):988-995. https://doi.org/10.1038/mp.2011.149

19. Schloß S, Ruhl I, Müller V, Becker K, Skoluda N, Nater UM, Pauli-Pott U (2018) Low hair cortisol concentration and emerging attention-deficit/hyperactivity symptoms in preschool age. Developmental Psychology 60:722-729. https://doi.org/10.1002/dev.21627

20. Susman EJ, Dockray S, Schiefelbein VL, Herwehe S, Heaton JA, Dorn LD (2007) Morningness/eveningness, morning-to-afternoon cortisol ratio, and antisocial behavior problems during puberty. Developmental Psychology 43 811-822. https://doi.org/10.1037/0012-1649.43.4.811

21. Blomqvist $M$, Holmberg K, Lindblad F, Fernell E, Ek U, Dahllöf G (2007) Salivary cortisol levels and dental anxiety in children with ADHD. Eur J Oral Sci 115:1-6. https://doi.org/10.1111/j.1600-0722.2007.00423.x

22. Ma L, Chen YH, Chen H, Liu YY, Wang YX (2011) The function of hypothalamus-pituitary-adrenal axis in children with ADHD. Brain Res 1368: 159-162. https://doi.org/10.1016/j.brainres.2010.10.045

23. Isaksson J, Nilsson KW, Lindblad F (2013) Early psychosocial adversity and cortisol levels in children with attention- deficit/ hyperactivity disorder. European Child and Adolescent Psychiatry 22:425-432. https://doi.org/10.1 007/s00787-013-0383-0

24. Hastings PD, Fortier I, Utendale WT, Simard LR, Robaey P (2009) Adrenocortical functioning in boys with attention-deficit/ hyperactivity disorder: examining subtypes of ADHD and associated comorbid conditions. Journal of Abnormal Child Psychology 37:565-578. https://doi.org/10.1007/s10802-008-9292-y

25. Meguid N, Reda M, Sheikh M et al (2016) Salivary cortisol levels in abused children with attention deficit hyperactivity disorder. J Psychiatry 19(01):348. https://doi.org/10.4172/2378-5756.1000348

26. Gray JA (1982) The neuropsychology of anxiety: an enquiry into the functions of the septohippocampal system. Oxford University Press, Oxford

27. Barkley RA (1997) Behavioral inhibition, sustained attention, and executive functions: constructing a unifying theory of ADHD. Psychol Bull 121:65-94. https://doi.org/10.1037/0033-2909.121.1.65

28. Sondeijker FE, Ferdinand RF, Oldehinkel AJ et al (2007) Disruptive behaviors and HPA-axis activity in young adolescent boys and girls from the general population. J Psychiatric Res 41:57-578 
29. Hatzinger M, Brand S, Perren S, von Wyl A, von Klitzing K, Holsboer-Trachsler E (2007) Hypothalamic-pituitary-adrenocortical (HPA) activity in kindergarten children: importance of gender and associations with behavioral/emotional difficulties. Journal of Psychiatric Research 41:861-870. https://doi.org/10.101 6/j.jpsychires.2006.07.012

30. Hirvikoski T, Lindholm T, Nordenstrom A et al (2009) High self-perceived stress and many stressors, but normal diurnal cortisol rhythm, in adults with ADHD (attentiondeficit/ hyperactivity disorder). Horm Behav 55(3):418-424. https://doi.org/10.1016/j.yhbeh.2008.12.004

31. Christiansen H, Oades RD, Psychogiou L, Hauffa BP, Sonuga-Barke EJ (2010) Does the cortisol response to stress mediate the link between expressed emotion and oppositional behavior in ADHD? Behav Brain Functions 6:45. https://doi.org/10.1186/1744-9081-6-45

32. Wang LJ, Huang YS, Hsiao CC, Chiang YL, Wu CC, Shang ZY, Chen CK (2011) Salivary dehydroepiandrosterone, but not cortisol, is associated with attention deficit hyperactivity disorder. The World Journal of Biological Psychiatry 12(2):99-109. https://doi.org/10.3109/15622975.2010.512090

33. Tsigos C, Chrousos GP (2002) Hypothalamic-pituitary-adrenal axis, neuroendocrine factors and stress. J Psychosom Res. 53(4):865-871. https:// doi.org/10.1016/S0022-3999(02)00429-4

34. Northover C, Thapar A, Langley K, Fairchild G, van Goozen SHM (2016) Cortisol levels at baseline and under stress in adolescent males with attention-deficit hyperactivity disorder, with or without comorbid conduct disorder. Psychiatry Res. 242:130-136. https://doi.org/10.1016/j.psychres.201 6.05 .052

35. Salis KL, Bernard K, Black SR, Dougherty LR, Klein D (2016) Examining the concurrent and longitudinal relationship between diurnal cortisol rhythms and conduct problems during childhood. Psychoneuroendocrinology 71: 147-154. https://doi.org/10.1016/j.psyneuen.2016.05.021

36. Işıka U, Bilgiç A, Toker A et al (2018) Serum levels of cortisol, dehydroepiandrosterone, and oxytocin in children with attention-deficit/ hyperactivity disorder combined presentation with and without comorbid conduct disorder. Psychiatry Research 261:212-219. https://doi.org/10.1016/j. psychres.2017.12.076

37. Freitag CM, Hanig S, Palmason H et al (2009) Cortisol awakening response in healthy children and children with ADHD: impact of comorbid disorders and psychosocial risk factors. Psychoneuroendocrinology 34(7):1019-1028. https://doi.org/10.1016/j.psyneuen.2009.01.018

38. Kaneko M, Hoshino Y, Hashimoto S, Okano T, Kumashiro H (1993) Hypothalamic-pituitaryadrenal axis function in children with attention-deficit hyperactivity disorder. J Autism Dev Disord 23(1):59-65. https://doi.org/10.1 007/BF01066418

39. Shoal GD, Giancola PR, Kirillova GP (2003) Salivary cortisol, personality, and aggressive behavior in adolescent boys: a 5-year longitudinal study. J Am Acad Child Adolesc Psychiatry 42:1101-1107. https://doi.org/10.1097/01.CHI. 0000070246.24125.6D

40. Lopez-Duran NL, Olson SL, Hajal NJ, Felt BT, Vazquez DM (2009) Hypothalamic pituitary adrenal axis functioning in reactive and proactive aggression in children. J Abnorm Child Psychol. 37:169-182. https://doi. org/10.1007/s10802-008-9263-3

41. Loney J, Carlson GA, Salisbury H, Volpe RJ (2005) Validation of three dimensions of childhood psychopathology in young clinic-referred boys. J Atten Disord. 8:169-181. https://doi.org/10.1177/1087054705279298

\section{Publisher's Note}

Springer Nature remains neutral with regard to jurisdictional claims in published maps and institutional affiliations.

\section{Submit your manuscript to a SpringerOpen ${ }^{\circ}$ journal and benefit from:}

- Convenient online submission

- Rigorous peer review

- Open access: articles freely available online

- High visibility within the field

- Retaining the copyright to your article

Submit your next manuscript at $\boldsymbol{\nabla}$ springeropen.com 